\title{
Web 2.0 e Pesquisa: Um Estudo do Google Docs em Métodos Quantitativos
}

Adriana Freire da Silva - UNEAL/Campus I - freiredriana@gmail.com

Dayvid Evandro da Silva Lós - UNEAL/Campus I - dayvid.faculdade@gmail.com

Djalma Rodolfo da Silva Lós - UNEAL/Campus I - rodolfo91@ gmail.com

Resumo. As abordagens inovadoras de ensino vêm se configurando como o meio mais eficiente de se alcançar uma educação de qualidade. Nesse contexto, as ferramentas da Web 2.0 aliam-se cada vez mais às práticas educativas a fim de alcançar seus objetivos. Entretanto, percebemos que nas pesquisas Survey poucas mudanças ocorreram nas práticas de gerenciar/administrar os questionários. Diante desse quadro, este trabalho busca realizar reflexões sobre a contribuição das ferramentas da Web 2.0, em especial o Google Docs, na pesquisa Survey, partindo de questionamentos acerca do uso dessa ferramenta mediando os processos de elaboração, disponibilização e avaliação dos questionários. Procuramos responder aos questionamentos a partir de um estudo bibliográfico e análises de duas formas de apresentação dos questionários, uma na forma do texto impresso e outra através do Google Docs. Percebemos que o uso dessa ferramenta possibilita ao pesquisador uma diversidade de estratégias como também uma economia coletiva nos processos do método de pesquisa.

Palavras-chaves: Web 2.0. Google Docs. Questionários

\section{Web 2.0 and Research: A Study of Quantitative Methods in Google Docs}

Abstract. The innovative teaching approaches are becoming increasingly the most efficient way to achieve a quality education. In this context, Web 2.0 tools combine with increasingly educational practices in order to achieve their goals. However, we realize that the few changes occurred Survey research practices to manage / administer the questionnaires. Against this background, this paper seeks to make reflections on the contribution of Web 2.0 tools, especially Google Docs, Survey research, from questions about the use of this tool mediating the processes of development, delivery and evaluation of questionnaires. We try to answer the questions from a bibliographical study and analysis of two forms of presentation of questionnaires, one in the form of printed text and the other through Google Docs. We realized that using this tool allows the researcher to a variety of strategies as well as a collective economy in the processes of research method.

Keywords: Web 2.0. Google Docs. Questionnaires

\section{Introdução}

A abordagem emergente ensino com pesquisa está cada vez mais frequente no nível superior de ensino. As universidades preocupam-se com a transição das abordagens conservadoras de ensino, baseadas na reprodução do conhecimento, para as inovadoras, baseadas na construção do conhecimento, uma vez que as primeiras se mostraram incapazes de promover uma formação educativa satisfatória para a sociedade vigente. Alguns autores como Pienta et al (2005) e Neubauer et al (2007) acrescentam que somente com a conjugação dos paradigmas inovadores, como as abordagens progressista e ensino com pesquisa, é que será possível atender às exigências da sociedade moderna e assim contribuir para a construção do conhecimento. 
Através delas, como afirma Silva et al (2002, p. 09) "os educadores serão levados a investigar novas metodologias de ensino que busquem a qualidade diante desta visão sistêmica de mundo", isto é, os educadores irão buscar recursos/ferramentas tecnológicos/as os quais sejam compatíveis com o público de alunos presentes nas instituições de ensino, tais como projetores multimídia, computadores, softwares, a Internet, e outros, que são frutos da atual sociedade marcada pelo acelerado desenvolvimento das tecnologias de comunicação e informação.

Dentro desse contexto, surgem as ferramentas da Web 2.0 para contribuírem como suporte aos educadores para que estes possam inovar suas práticas pedagógicas e assim atingir os objetivos das abordagens inovadoras: transformar o ensino em um ambiente no qual o conhecimento vai ser construído por ações conjuntas entre aluno aluno e aluno - professor.

Essas ferramentas presentes essencialmente na Internet podem mediar esse espaço auxiliando de forma efetiva em vários tipos de pesquisa, sejam elas bibliográficas ou experimentais. Ainda mais, essas ferramentas "constituem uma oportunidade para que professores e alunos possam aprender colaborativamente, divulgando e compartilhando as suas experiências e saberes" acrescenta Carvalho (2008, p. 13). Percebemos, então, que a partir do uso dos recursos que a tecnologia proporciona, o ensino passa a ser visto de forma diferente, há um espaço de interação, antes impossibilitado pelas diferenciações impostas entre professor e aluno.

No que se refere à pesquisa, na vida acadêmica professores e alunos precisam desenvolvê-la seja durante a graduação, mestrado, doutorado, com o propósito de atender as exigências do paradigma emergente: ensino com pesquisa, ou até mesmo para aprovação nos graus citados através de artigos, monografias, dissertações, teses e outros. Percebemos, entretanto, que nas pesquisas do tipo quantitativa baseadas em questionários ou entrevistas (Survey), mesmo com o crescente uso de tecnologias pelas instituições de ensino, poucas mudanças ocorreram na elaboração, disponibilização e avaliação desses questionários. É feito o uso de papel com os questionamentos escritos ou digitados, e somente no tratamento dos dados coletados que se emprega algum tipo de tecnologia diferenciada. Dessa forma, o uso superficial das tecnologias prevalece e passamos a perceber que poucas mudanças aconteceram, a não ser a transição do manual para o digital com relação à elaboração dos questionários.

Assim, alguns questionamentos são levantados acerca do uso de questionários em pesquisas Survey: Os questionários podem ser elaborados diferentemente do comum, como por exemplo, com o uso das ferramentas da Web 2.0? Que vantagens são possibilitadas com elaboração e disponibilização de questionários por meio de uma ferramenta da Web 2.0? Quando será pertinente o uso de questionário comum (impresso) ou o gerenciado por alguma ferramenta da Web 2.0?

Para respondermos tais questionamentos, realizamos duas análises de questionário, uma empregada tradicionalmente nas mais diversas pesquisas quantitativas - questionário impresso - e a outra mediada por uma ferramenta da Web 2.0, o Google Docs, selecionada para elaboração, disponibilização e avaliação dos questionários.

Tais análises são fundamentadas por conceitos de pesquisa fazendo referência aos métodos, principalmente o quantitativo, e por reflexões acerca da Web 2.0 fazendo referência as suas ferramentas, destacando o Google Docs.

Assim, o trabalho é composto pelos conceitos e análises citados, e por fim as considerações finais nas quais buscamos responder aos questionamentos apresentados como também argumentamos a importância do trabalho para a comunidade acadêmica. 


\section{Conceitos}

\subsection{Conceituando Pesquisa}

$\mathrm{Na}$ vida acadêmica é essencial o ato de se fazer pesquisa, não somente para cumprir o quadro exigido pelas instituições de ensino, mas também da necessidade humana de buscar compreender todas as relações que são estabelecidas entre os seres no mundo. Assim, fazer pesquisa surge da necessidade de buscar respostas a indagações determinadas, como aponta Gil (2002, p. 17) "a pesquisa é requerida quando não se dispõe de informação suficiente para responder ao problema, ou então quando a informação disponível se encontra em tal desordem que não possa ser adequadamente relacionada ao problema". Esse processo tem como resultado a construção de um saber/conhecimento através do confronto de dados, informações coletadas e conhecimentos teóricos acumulados a respeito do que é estudado.

Complementam Lüdke e André (1986) que esse conhecimento construído não é somente fruto da curiosidade, da inquietação, da inteligência e da atividade investigativa do pesquisador. Há também o resgate do que já foi estudado por vários pesquisadores acerca de determinado tema/problemática a fim de promover uma continuação, um avanço de teorias de acordo com as mudanças na sociedade e na ciência.

No entanto, construir/produzir conhecimento não se dá de forma desordenada, ao acaso. São necessários planejamento e sistematização das etapas que levam a sustentar/validar esse conhecimento. A partir disso, podemos definir pesquisa de acordo com Gil (2002, p. 17), para ele "é um processo racional e sistemático [...] desenvolvida mediante o concurso dos conhecimentos disponíveis e a utilização cuidadosa de métodos, técnicas ou procedimentos científicos".

Observamos a ênfase no término da citação de Gil (2002) quando trata dos métodos de pesquisa. Realmente, essa etapa é de fundamental importância para a pesquisa já que é através dela que os dados são coletados, estruturados e analisados com o intuito de responder aos questionamentos da pesquisa. Além disso, um planejamento antecipado é necessário para suprir e orientar o andamento da pesquisa, no qual também está presente a opção dos métodos de pesquisa.

Enfatizamos neste trabalho os métodos e técnicas de pesquisa, especialmente o Survey, que podem auxiliar o pesquisador na coleta dos dados, estes imprescindíveis para o desenvolvimento sustentável da pesquisa uma vez que são vitais para que uma pesquisa seja eficiente e dessa forma cumpra com o seu papel: a construção e a consolidação do conhecimento.

\subsection{Método de Pesquisa Survey}

Como nosso objeto de estudo são os questionários acadêmicos, aqueles responsáveis por coletar dados em pesquisas quantitativas, especificamente a Survey, achamos pertinente entender esse método de pesquisa conceitualmente.

Para Tanur (1982) citado por Pinsonneault e Kraemer (1993) a pesquisa Survey é a aquisição de dados ou informações sobre características, ações ou opiniões de um grupo de pessoas selecionadas (público visado), por meio de um instrumento de pesquisa, normalmente o questionário. Ou seja, tem como objetivo fazer descrições quantitativas acerca de uma população através de um instrumento predefinido.

Essa predefinição é necessária visto que nesse tipo de pesquisa pretende-se estudar algo que está acontecendo ou que aconteceu recentemente, ou seja, o pesquisador pode presumir uma série de premissas/hipóteses e através do questionário confirmá- las ou inferir outras situações válidas para a pesquisa.

Por isso, é recomendada a prática dessa metodologia quando: o pesquisador busca responder às perguntas, “o que?", "por que?", “como?” e "quanto?”, isto é, o 
importante é entender como e porque algo está acontecendo; não há interesse ou não é possível controlar as variáveis dependentes e independentes, uma vez que a manipulação dos dados advindos dos questionários é dada por igual, única, não há necessidade de controlar as condições observadas para testar a hipótese formulada como é o caso do método experimental; quando o ambiente estiver em seu modo natural, sem estratégias de modificação, assim é possível estudar o fenômeno de forma mais objetiva, com maior precisão (FINK 1995a; 1995b).

Percebemos, então, que nesse tipo de pesquisa os questionários são essenciais para o investigador. É através deles que as informações relevantes para a pesquisa são extraídas, analisadas através de alguma ferramenta e consolidadas por meio da publicação da pesquisa.

\subsection{Conceituando Web 2.0}

De forma geral, os métodos e técnicas quando bem selecionados tornam a pesquisa bem sucedida, isto é, o pesquisador através dessa seleção e consequentes análises, consegue atender aos objetivos da pesquisa de forma eficiente e clara. Entretanto, durante tal escolha, é preciso se atentar aos gastos decorrentes da realização de cada passo da metodologia, sejam eles, humanos, materiais ou financeiros, Gil (2002). Ou seja, os investigadores devem buscar meios os quais além de proporcionar uma melhor obtenção dos dados também sejam viáveis economicamente.

Com os avanços tecnológicos dos últimos anos, a sociedade passou por diversas mudanças de efeito global. São mudanças relacionadas a diversas esferas, como as relações familiares, o convívio humano, o trabalho e a educação. Nesta última, várias ferramentas da $W e b$ vêm surgindo e podem auxiliar na prática de pesquisa acadêmica especialmente. Essas ferramentas possibilitam uma série de recursos jamais disponibilizados em outros tempos, proporcionando ao pesquisador uma diversidade de estratégias que podem ser tomadas a qualquer momento. Assim, tais recursos passam a contribuir significativamente no processo de pesquisa e consequentemente em sua eficiência.

Essas ferramentas da Web são resultado de uma mudança de filosofia da Internet. Em um primeiro momento, era tida como um local de leitura de páginas da $W e b$ as quais eram programadas por técnicos específicos, ou seja, o usuário comum não podia inserir, comentar, editar conteúdos, era literalmente um espectador. Em um segundo momento, a Internet tornou-se um espaço em que todos os participantes são ativos, isto é, o usuário comum pode enviar, editar, comentar, alterar, excluir conteúdos de forma simples e fácil. A essa transição é o que O'Reilly (2005) nomeia de Web 2.0, que pode referir-se a várias situações como Primo (2006, p. 01) discorre

Web 2.0 pode referir-se a uma combinação de técnicas informáticas (serviços Web, linguagem Ajax, Web syndication, etc.), a um momento histórico, a um conjunto de novas estratégias mercadológicas para o comércio eletrônico e a processos de interação social mediados pelo computador.

Em nossas análises vamos enfatizar a última instância citada por Primo (2006) quando argumenta que a Web 2.0 proporciona um espaço de interação entre usuários mediados por um computador ou qualquer outro equipamento conectado à internet, não desconsiderando as relações entre todas as instâncias citadas.

Um dos princípios fundamentais da Web 2.0 é o que O'Reilly (2005) aborda: o tratamento da Internet como uma plataforma, isto é, meio (espaço) no qual é possível a promoção de serviços que anteriormente só poderiam ser geridos através de softwares 
instalados em computadores. Além disso, dá aos sites características de interação entre usuários, jamais estabelecidas nos meios virtuais (PRIMO, 2006).

Esses serviços online também são conhecidos como as ferramentas da Web 2.0. Várias delas estão disponíveis na Internet para auxiliarem o usuário em alguma tarefa. São algumas bastante difundidas na Internet: Blogue, Youtube, Delicious, Wikipedia, Mapas Conceituais Online e Google Docs.

Há um uso expressivo dessas ferramentas pelo público virtual uma vez que uma das principais características da Web 2.0 é a de criar interfaces amigáveis possibilitando para o usuário uma fácil adaptação que reflete em uma rápida aprendizagem no uso da ferramenta, Machado (2009). Outro fator bastante influenciador é a gratuidade da maioria das ferramentas. Qualquer usuário com apenas alguns cliques do mouse poderá usufruir dos recursos que os serviços online podem oferecer.

Dentre as ferramentas da Web 2.0 citadas acima, vimos o Google Docs. Esta é uma ferramenta que vem aos poucos se manifestando com grande potencial de se aliar à educação, como é o caso da abordagem ensino com pesquisa. Surgiu em 2006, através da unificação de dois serviços, o de processamento de textos e de planilhas. Podemos defini-la atualmente como o conjunto de serviços online capazes de processar textos, planilhas, apresentações, desenhos e formulários de forma colaborativa e gratuita, isto é, vários usuários podem estar participando ativamente do processo de criação e edição de tais documentos.

Optamos por essa ferramenta, pois através de seu serviço formulário possibilita ao investigador formas inovadoras de elaboração, disponibilização e avaliação dos questionários tidos em pesquisas Survey.

\section{Análises}

\subsection{Como o questionário é tido geralmente nas pesquisas}

Como já foi dito anteriormente, a prática de questionários durante as pesquisas quantitativas é muito empregada pelos pesquisadores de qualquer área do conhecimento. Limitamos neste momento a fazer análises de como esse questionário é elaborado, disponibilizado/aplicado e de como é feito o processo de avaliação.

Os questionários são elaborados pelos pesquisadores manualmente, através de papel, caneta, lápis, entre outras tecnologias tradicionais ou através de computadores com a digitação em um software editor de texto ou de planilha e a impressão dos mesmos. Em ambos os casos há o processo de cópia. A quantidade irá depender do grupo/população visado.

Para efetivar a pesquisa quantitativa, os pesquisadores em sua maioria precisam passar pelas seguintes etapas: escrever ou digitar o questionário, realizar a impressão ou o processo de cópia a depender do grupo/população visado, ir ao local onde permanece esse grupo/população (escola, universidade, hospital, população de uma cidade, etc.), aplicar o questionário informando as devidas orientações, aguardar o público visado responder aos questionários e por fim fazer a sua recolha. Após esse processo inicial, o pesquisador ainda usaria algum equipamento tecnológico, no caso o computador, para transferir essas informações para um dispositivo de armazenamento de modo a torná-las seguras para possíveis análises.

Discutimos abaixo a elaboração, disponibilização e avaliação dos questionários interpretados a partir das etapas citadas anteriormente.

i. Os questionários passam a ser digitados ao invés de escritos;

Percebemos que houve uma mudança apenas de suporte de elaboração, ou seja, as atividades que moldam são as mesmas, não houve uma mudança efetiva de 
elaboração que promovesse efeitos diferenciados/inovadores em sua aplicação, e, portanto, na pesquisa.

ii. Durante a aplicação dos questionários, é necessário alguém no local que está presente o grupo/população visado;

iii. Cópias ou impressões serão realizadas para atender o grupo/população visado. A quantidade irá depender dos objetivos da pesquisa;

iv. Tempo prolongado para ir ao local, explanar o questionário, aguardar e orientar o seu preenchimento, e realizar a sua recolha;

Observamos de acordo com os itens listados que há uma grande dependência no passo a passo dos procedimentos até a conclusão da etapa de disponibilização, isto é, os procedimentos só acontecem por meio de um condutor humano. Essa dependência acentuada necessita por parte do pesquisador de um maior planejamento para realização da pesquisa, que por sua vez irá acarretar em uma exigência de tempo maior para concluir a etapa de disponibilização/aplicação dos questionários. Outro fator considerável é o de obrigatoriedade de cópias, ou seja, a única forma de coletar os dados é distribuindo os questionários impressos ao público visado, aguardar o devido preenchimento e por fim recolhê-los.

v. Necessidade de transferir os dados coletados através do questionário impresso para um dispositivo de armazenamento geralmente mediado por um computador;

vi. Impossibilidade de observar em tempo real a coleta dos dados a partir dos questionários;

Com relação à avaliação dos questionários já devidamente preenchidos pelo grupo/população visado, notamos de imediato, a necessidade de transferir as informações para um computador e logo serem tratadas em um software editor de textos ou de planilhas, como por exemplo, o Microsoft Office ou LibreOffice (BrOffice). Isso para que as análises sejam ágeis, seguras e eficientes, seja por meio de um agrupamento de dados, da elaboração de gráficos, de algum filtro, enfim, um tratamento de dados que vai depender dos objetivos do pesquisador e da sua consequente pesquisa. Notamos, ainda nesta etapa, a alta dependência do fator humano como intermediador dos processos.

A impossibilidade de observar a coleta dos dados em tempo real através desse tipo de questionário também é logo observada. Somente é possível obter informações relevantes através do questionário após o seu término, isto é, depois que realizar todos os procedimentos das etapas anteriores discutidas. O pesquisador pode insistir em ter informações antes do término da aplicação dos questionários, porém, isso acentuaria ainda mais o fator tempo durante os processos, já que realizaria a etapa (v) uma ou várias vezes, a depender dele. Ainda assim, a análise nunca seria em tempo real.

vii. Diminuição da precisão/confiabilidade dos dados durante os processos.

Notamos uma diversidade de procedimentos para finalizar as etapas de pesquisa quantitativa através desse tipo de questionário, desde a elaboração à aplicação e recolha dos questionários. Essa diversidade complementada pelo fator humano que vem sendo destacado nas análises, até o momento, diminui a precisão e confiabilidade dos dados presentes nos questionários, já que há uma grande probabilidade de falha entre uma ou outra etapa, principalmente no ato da recolha do questionário e logo na de transmissão dos dados para um software em um computador. Assim, esse fator pode fazer com que a 
credibilidade da pesquisa seja reduzida, prejudicando todo um trabalho planejado e sistematizado pelo pesquisador.

\subsection{O questionário baseado em uma ferramenta da Web 2.0: o Google Docs}

A partir da descrição das análises realizadas sobre como o questionário ainda é tido nas pesquisas quantitativas nos meios acadêmicos, especificamente do tipo Survey, é que nos motivamos a demonstrar os procedimentos de elaboração, disponibilização e avaliação seguindo o mesmo raciocínio, porém, sob outra plataforma, em uma ferramenta da Web 2.0, o Google Docs.

É importante destacar que esta discussão se remete apenas as análises dos questionários, ou seja, nos efeitos que tal ferramenta pode proporcionar como administradora dos formulários ${ }^{1}$ referenciando as pesquisas acadêmicas do tipo Survey. A forma de manusear detalhadamente os formulários no Google Docs não é o foco do trabalho, visto que há vários documentos - tutoriais, manuais, vídeos, - postados na Internet, principalmente no site do desenvolvedor da ferramenta, que fazem esse tipo de orientação.

Os serviços do Google Docs que são responsáveis pelo gerenciamento de documentos, apresentações, desenhos, e outras opções de formulário também não serão discutidos. Este último pode conter intuitos/objetivos diferentes dos quais tomamos neste trabalho - coletar dados em uma pesquisa quantitativa - como, por exemplo, cadastro de eventos para diversas finalidades.

Antes de dar início a elaboração do questionário no Google Docs, é preciso ter um e-mail e acessar o Google Docs através do link ${ }^{2}$, http://docs.google.com, em algum browser (navegador de internet) inserindo os dados da conta para realizar o Login ${ }^{3}$. Após isso, o Google Docs está pronto para elaboração do questionário.

Abaixo, discutimos os procedimentos de elaboração, disponibilização e avaliação dos questionários tendo como mediador o Google Docs.

i. Os questionários passam a ser gerenciados pelo Google Docs ao invés de digitados em um programa editor de textos ou de planilhas;

Diferentemente da forma de questionário discutida anteriormente, a elaboração neste caso tem uma mudança notável, generalizada. As perguntas do questionário são inseridas diretamente no Google Docs, na ferramenta "formulários" e ficam disponíveis online já que o formulário está sendo salvo em um servidor virtual da Google. É nesta etapa que o pesquisador vai orientar o público visado sobre como responder aos questionamentos. Por isso, é a etapa que o pesquisador irá precisar de um maior tempo uma vez que irá exigir um bom planejamento e uso de estratégias que irão depender do público que irá ter acesso ao formulário em sua futura disponibilização.

Dessa vez, não houve apenas uma mudança de suporte, mas sim de toda a atividade de gerenciamento dos questionários, justamente pelo fato de estar online, disponível a qualquer momento, e estar sendo administrado pelo Google Docs, o qual traz consigo uma bagagem de recursos que possibilitam estratégias de análise pelo pesquisador impossíveis até então. Tais recursos refletem na disponibilização e avaliação dos questionários, que é o que vamos discutir a seguir. Verificamos adiante alguns resultados do uso de tais recursos.

ii. Após gerar o link do formulário e enviá-lo para o público visado, não há necessidade de encontros presenciais para a aplicação do questionário; 
iii. Não há uso de papel na aplicação dos questionários já que o formulário é respondido/preenchido online por meio de computadores ou equipamentos conectados à internet;

Após a elaboração do questionário na ferramenta Google Docs, a tarefa se torna muito simples para atingir o público visado. A disponibilização do questionário é dada através da Internet por meio de um link, como este, https://spreadsheets.google.com/viewform?formkey=dGtUQkVnbEM1Z2FxYmhRTENMTjRO bW6MQ, gerado automaticamente pela ferramenta. Não é necessário, portanto, o deslocamento do pesquisador para o local onde está presente o grupo/população visado, em contrapartida, é imprescindível que esse grupo a ser questionado tenha acesso a computadores ou outros equipamentos conectados à internet. Basta, pois, enviar o link do formulário para o público visado, seja via e-mail, SMS, Facebook, Orkut, Twitter e outros. Como consequência, não é necessária a impressão do questionário ou realização de cópias, isto é, fica dispensada a utilização de papel na aplicação do questionário, visto que o formulário é acessado e respondido através da Internet.

iv. Os dados inseridos pelo público-alvo no formulário online são vinculados a uma planilha eletrônica;

v. A qualquer momento pode ser observado como está o andamento da pesquisa, ou seja, a análise pode ser realizada em tempo real;

No momento em que a etapa de envio/disponibilização do link, que leva o usuário a responder ao formulário/questionário, estiver concluída, o pesquisador somente irá aguardar o grupo visado iniciar o envio de informações requisitadas no formulário. Essas informações são imediatamente vinculadas a uma planilha semelhante a do software Microsoft Office Excel, sendo diferentes com relação à quantidade de recursos oferecidos. Essa semelhança possibilita uma familiarização do pesquisador para com a ferramenta, gerando assim uma rápida adaptação e consequentemente um melhor uso da ferramenta refletindo em uma maior produtividade.

Dessa forma, não há necessidade de realizar a transferência de dados como na análise do questionário anterior, é possível exportar a planilha para um arquivo (.xls) para melhor ser analisada/tratada em uma ferramenta mais completa, como por exemplo o Microsoft Office Excel ou LibreOffice Calc.

O pesquisador ou grupo designado que possuir a conta da Google e sua senha poderá observar o andamento da pesquisa em tempo real, independente do local em que esteja. É possível ainda convidar pessoas específicas para que tenham acesso a essa planilha, ou seja, indivíduos com a devida permissão poderão observar as respostas e a qualquer momento há a possibilidade de realizar algum tipo de edição, seja removendo ou acrescentando algum item no formulário. Tudo de forma ágil e simples através de algum equipamento conectado à internet.

vi. O procedimento é único por parte do público-alvo: somente acessar o link do formulário e inserir os dados, assim, os dados da pesquisa se tornam mais confiáveis/precisos;

vii. Os dados não são armazenados em dispositivos físicos, mas sim em servidores virtuais, neste caso, o da Google.

Vimos na análise do questionário anterior que devido à quantidade de etapas de elaboração, disponibilização e avaliação os dados teriam certa probabilidade de diminuir a confiabilidade e precisão. Desta vez isso minimiza bastante, uma vez que o público visado insere as informações diretamente na planilha por meio do formulário, ou seja, o procedimento é único, não há transporte de dados por contato humano. A 
partir disso, é gerada uma maior credibilidade para a pesquisa como também uma maior segurança/confiabilidade na análise dos dados. Tratando de segurança, todos os dados inseridos no formulário são armazenados em servidores virtuais, e não em dispositivos físicos como pendrive ou disco rígido. Acentua, neste caso, a segurança das informações e como consequência a da pesquisa, visto que os dados coletados são valiosos para o pesquisador. É de se atentar, no entanto, às vulnerabilidades que existem nos meios virtuais, como possíveis falhas nos servidores, perdas de credenciamento, manutenções dos serviços, entre outras.

\section{Considerações Finais}

A partir da leitura das discussões acerca das duas formas de elaborar, disponibilizar e avaliar os questionários, uma tida geralmente nas pesquisas acadêmicas, a outra fundamentada em uma ferramenta da Web 2.0, o Google Docs, percebemos algo bastante notável e considerável: a economia, isto é, a transição dos questionários passa a produzir uma economia coletiva, relacionada, por exemplo, a recursos financeiros que passam a ser menores, ao menor desgaste humano nos processos, a menor preocupação do tempo no planejamento, além de possíveis imprevistos que poderiam acontecer na primeira análise discutida devido à quantidade de procedimentos realizados em cada etapa.

A diversidade de características vantajosas que o Google Docs possibilita como administrador dos formulários/questionários é evidente, já que ele está em um meio favorável que é a Internet. Ratifica a ideia de Web 2.0 na qual a Internet é tida como um espaço compartilhado entre usuários em que estes podem criar, inserir, editar, compartilhar, comentar conteúdos, o que em outros tempos não era possível.

No entanto, é preciso destacar ainda que nosso objetivo com este trabalho não é o de intensificar a substituição das formas de administrar/gerenciar os questionários, diferentemente, discutimos algumas particularidades de ambas as formas com o intuito de possibilitar ao leitor o conhecimento de que existem outras formas além das quais geralmente são expostas na escola ou na universidade, como foi o caso do uso do Google Docs. Além disso, a depender dos objetivos da pesquisa e do consequente público alvo, não vai ser possível trabalhar com o Google Docs, seja por fatores relativos a taxa de retorno, que pode ser mínima ou por uma ausência de uma melhor orientação a respeito das perguntas, ou seja, é pertinente o uso de uma ou outra forma de questionário ou até mesmo de ambas as formas, tudo a depender do pesquisador e seus objetivos com relação a pesquisa.

Acreditamos que os paradigmas emergentes da educação cada vez mais se aliam ao uso das tecnologias e novos rumos são criados e possibilitados de serem atingidos. É preciso, entretanto, como argumenta Carvalho (2008) que professores e alunos em um primeiro momento conheçam as potencialidades e funcionalidades da ferramenta a qual pretende utilizar e logo depois façam uso da aprendizagem em alguma prática acadêmica.

Neste trabalho enfatizamos o uso de uma ferramenta da Web 2.0, o Google Docs, em um contexto de pesquisa acadêmica, especificamente o método de pesquisa Survey. É de caráter inicial o trabalho, uma vez que pretendemos realizar outros estudos com a ferramenta Google Docs a fim de melhor entender as opções pedagógicas que ela pode proporcionar.

\footnotetext{
${ }^{1} \mathrm{O}$ termo questionário a partir desse momento assemelha-se a função de formulário no Google Docs. Ou seja, quando citamos o termo formulário estamos tratando de questionário. Ocorre esta mudança vis to que os suportes são diferentes, questionário sob suporte papel impresso e formulário sob suporte tecnológico.
} 
${ }^{2} \mathrm{O}$ termo Link significa ligação, isto é, é uma referência num documento em hipertexto a outras partes deste documento ou a outro documento.

${ }^{3}$ Em termos informáticos, Login define o processo através do qual o acesso a um sistema informático é controlado através da identificação e autenticação do utilizador através de credenciais fornecidas por esse mesmo utilizador, por exemplo o e-mail e sua senha. Conforme Wikipédia: http://pt.wikipedia.org/wiki/Login

\section{Referências Bibliográficas}

CARVAlHO, A. A. Manual de Ferramentas da Web 2.0 para Professores. Lisboa: DGIDC, Ministério da Educação. 2008. Disponível em: 〈http://www.crie.min-edu.pt>. Acesso em: 10 jul. 2011.

FINK, Arlene. The survey handbook. Thousand Oaks, Sage, 1995a.

FINK, Arlene. How to design surveys. Thousand Oaks, Sage, 1995b.

GIL, Antonio Carlos. Como elaborar projetos de pesquisa. $4^{\mathrm{a}}$ ed. São Paulo: Atlas, 2002.

LÜDKE, Menga; ANDRÉ, Marli E.D.A. PESQUISA EM EDUCAÇÃO: ABORDAGENS QUALITATIVAS. São Paulo: EPU, 1986.

MACHADO, Ana Claudia Teixeira. A FERRAMENTA GOOGLE DOCS: CONSTRUÇÃO DO CONHECIMENTO ATRAVÉS DA INTERAÇÃO E COLABORAÇÃO. Paideia, v. 2, n. 1, jun. 2009. ISSN 1982-6109.

NEUBAUER, Airton; ALMEIDA, Siderly do Carmo Dahle de; ASSAD, Ricardo. UM OLHAR SOBRE A TRAJETÓRIA DA EDUCAÇÃO: DOS PARADIGMAS CONSERVADORES AO PARADIGMA EMERGENTE. Athena - Revista Científica de educação, v. $8, \quad$ n. 8 , jan./jun. 2007. Disponível em: <www.faculdadeexpoente.edu.br>. Acesso em: 18 jun. 2011.

O'Reilly, Tim. "What Is Web 2.0 - Design Patterns and Business Models for the Next Generation of Software." O'Reilly Network: What is Web 2.0. 30 Sept 2005. O'Reilly Publishing. 02 Apr. 2011.

PINSONNEAULT, A. e KRAEMER, K. L. Survey research in management information systems: an assessement. Journal of Management Information System, 1993.

PIENTA, Ana Cristina Gipiela; BERTICELLI, Danilene Donin; GASPAR, Maria Dagmar da Rocha; BEHRENS, Marilda Aparecida. EDUCAÇÃO, FORMAÇÃO PROFISSIONAL DOCENTE E OS PARADIGMAS DA CIÊNCIA. Revista Olhar de Professor, v. 8, n. 2. Universidade Estadual de Ponta Grosa, Brasil. p. 93-106. 2005. ISSN: $1518-5648$.

PRIMO, Alex. O aspecto relacional das interações na Web 2.0. In: Intercom 2006 XXIX Congresso Brasileiro de Ciências da Comunicação, UnB, 2006. Disponível em: <http $/ /$ www.intercom.org.br>. Acesso em: 04 jul. 2011.

SILVA, Ester Framarin de S; RAYMUNDO, Gisele Pontaroli; BEHRENS, Marilda Aparecida. PARADIGMAS CONTEMPORÂNEOS E A EDUCAÇÃO SUPERIOR. Revista Diálogo Educacional, v. 3, n. 5, p. 87-103, jan./abril. 2002. ISSN: 1518-3483. 